\title{
EL GÉNERO LYSAPHIDUS (HYMENOPTERA: BRACONIDAE) EN LA PENÍNSULA IBÉRICA
}

\author{
J. M. Michelena Saval (*) y A. Sanchis Segovia (*)
}

\begin{abstract}
RESUMEN
Se detecta la presencia del género Lysaphidus Smith, 1944 (Hymenoptera, Braconidae, Aphidiinae) en la Península Ibérica, representado por dos especies, $L$. arvensis Stary, 1960, nueva cita para la Peninsula Ibérica y L. santolinae n. sp., parasitoides ambos del género Coloradoa Wilson, 1910 (Homoptera, Aphididae), cuyas momias fueron recolectadas sobre Santolina chamaecyparissus L. y Santolina rosmarinifolia L. (Asteraceae).
\end{abstract}

Palabras clave: Parasitoides, Braconidae, Aphidiinae, nueva especie, Península Ibérica

\section{ABSTRACT}

The genus Lysaphidus (Hymenoptera: Braconidae) in the Iberian Peninsula

The genus Lysaphidus Smith, 1944 (Hymenoptera, Braconidae, Aphidiinae) is recorded for the first time from the Iberian Peninsula represented by two species: L. arvensis Stary, 1960, first record for the Iberian Peninsula, and L. santolinae n. sp., both of them parasitoids of Coloradoa Wilson,1910 genus (Homoptera, Aphididae). The mummies were collected on Santolina chamaecyparissus L. and Santolina rosmarinifolia L. (Asteraceae).

Key words: Aphid parasitoids, Braconidae, Aphidiinae, new species, Iberian Peninsula

\section{Introducción}

El género Lysaphidus Smith, 1944 fue descrito como un subgénero neártico de Aphidius Nees, estando representado por cuatro especies: L. adelocarinus Smith, 1944, L. multiarticulatus (Ashmead, 1888), L. ramithyrus Smith, 1944, L. rosaphidis Smith, 1944. En 1960, Stary reconoce la presencia de este género en Europa. En la actualidad son diez las especies adscritas a este género (Mackauer, 1968), de cuales L. arvensis Stary, 1960, L. erysimi Stary, 1960 y L. viaticus Sedlag, 1959, se encuentran en Europa.
Este género ha sido hallado por primera vez en la Península Ibérica, estando representado por dos especies. En el presente trabajo se describe una nueva especie y se cita L.arvensis Stary, 1960 por primera vez en la Península Ibérica.

Lysaphidus arvensis Stary, 1960

Lysaphidus arvensis Stary, 1960, Polskie Pismo ent., 30: 359361; holotype: H, Kostelní Lhota near Sadská, Bohemia/Czechoslovakia (Col. Stary).

* Departamento de Biología Animal, Facultad de Ciencias Biológicas, Universidad de Valencia. Dr. Moliner, 50. 46100 Burjassot-Valencia. 
Hospedadores: Coloradoa bournieri Remaudière \& Leclant, 1969 sobre Santolina chamaeciparissus L. en La Torre (Valencia): 9-6-93; La Yesa (Valencia): 6-6-95, 8-6-95, 14-6-95, 28-695; Puebla de San Miguel (Valencia): 19-5-93, 3-693, 10-6-93. Coloradoa moralesi Remaudière \& Leclant, 1969 sobre Santolina rosmarinifolia L. en Viniegra de la Sierra (Rioja): 13-6-93, 14-6-93, 47-95.

Distribución general: región Paleártica (Checoslovaquia, Francia, España).

\section{Lysaphidus santolinae $\mathrm{n} . \mathrm{sp}$.}

Material. Holotipo, $1 \mathrm{H}$, Puebla de San Miguel (ValenciaEspaña): 19-V-93. Paratipos: $2 \mathrm{H}$, Puebla de San Miguel (Valencia-España) : 19-V-93 y 2 M, Puebla de San Miguel (Valencia-España): 3-VI-93. El material examinado se encuentra depositado en la "Colección del Departamento de Biología Animal de la Universitat de València (España).

Hembra (Fig. 1). Cabeza ligeramente transversa, más ancha que el tórax a nivel de las tégulas, lisa, brillante, con pelos dispersos. Occipucio con reborde. Clípeo oval, ligeramente convexo, liso, brillante, con el borde inferior arqueado y presentando un reborde frontal. El índice tentorial es 0.27. Los ojos son grandes, ovales, ligeramente convergentes a nivel del clípeo. Antenas de 12 artejos, aunque algunos ejem- plares tienen 13, filiformes, ligeramente engrosadas hacia el ápice. Flagelómero 1 y 2, aproximadamente de igual longitud (en ocasiones el flagelo 1 ligeramente mayor), algo más de 3 veces más largos que la anchura de sus ápices. Diámetro de la foseta antenal, aproximadamente igual a la distancia entre ésta y la órbita ocular.

Tórax liso y brillante. Mesoscutum con notaulices evidentes sólo en la parte anterior, ligeramente crenulados. Propódeo presentando 2 quillas transversas, en la parte inferior, algo curvadas y sinuosas, que no delimita una areola central pentagonal (fig.1a). Ala anterior: pterostigma más de tres veces más largo que ancho; metacarpo algo menos de 1/3 más corto que la longitud del pterostigma; radial abcisa 1 algo más larga que la anchura del pterostigma.

Metasoma. Longitud del terguito 1 más de tres veces mayor que su anchura a nivel de los espiráculos, algo dilatado en el ápice; presentando ligeras impresiones laterales por detrás de los espiráculos, casi liso y con pelos aislados. Tubérculos espiraculares situados por delante de la mitad del segmento. Genitalia (fig. 1b).

Coloración. Cabeza castaño oscuro; cara, clípeo, piezas bucales y mejillas amarillos. Antenas castañas, con F1 y F2 amarillo-rojizos. Tórax castaño oscuro, excepto el protórax que es amarillo. Patas amarillas, presentando los ápices de los tarsos, los fémures (medios y posteriores) y las tibias
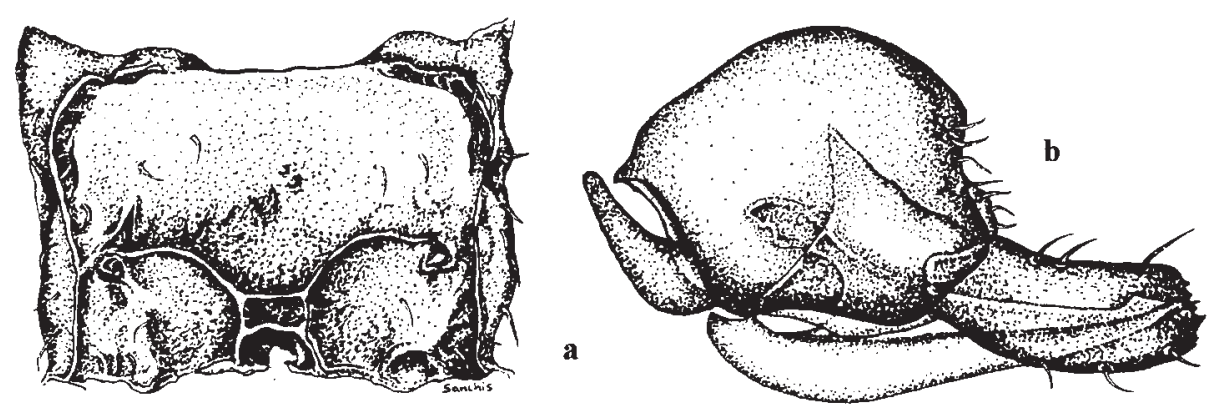

Fig. 1.- Lysaphidus santolinae n. sp. a, propódeo; b, genitalia hembra.

Fig. 1.-Lysaphidus santolinae n. sp. a, propodeum; b, female genitalia. 
oscurecidos. Metasoma amarillento en su inicio, castaño oscuro en su parte media, tendiendo al castaño en su tramo final. Terguito 1 amarillo. Valvas de la genitalia castaño oscuro.

Medidas. Longitud corporal: 1.31-1.65 mm; anchura cabeza: $0.31 \mathrm{~mm}$; distancia interocular: $0.18 \mathrm{~mm}$; línea facial: $0.13 \mathrm{~mm}$; distancia transverso-facial: $0.11 \mathrm{~mm}$; diámetro longitudinal del ojo: $0.13 \mathrm{~mm}$; distancia intertentorial: $62 \mathrm{~m}$; distancia tentorio-ocular: $20 \mathrm{~m}$; ancho en tégulas: $0.21 \mathrm{~mm}$; longitud del pterostigma: $0.24-0.33 \mathrm{~mm}$; ancho pterostigma: 0.07-0.09 mm; metacarpo: 0.20-0.24 mm; abcisa 1 de la radial: $0.09-0.11 \mathrm{~mm}$; abcisa 2 de la radial: $0.06-0.11 \mathrm{~mm}$; cubital: $0.11-0.14 \mathrm{~mm}$; interradial 2: $0.05-0.08 \mathrm{~mm}$; ancho a nivel de los espiraculos: $0.07 \mathrm{~mm}$; longitud terguito $1: 0.25 \mathrm{~mm}$.

Macho. Antenas de 15 artejos. Otras características similares a la hembra, excepto la coloración. Cabeza castaño oscuro con piezas bucales y clípeo más claros. Tórax castaño oscuro. Patas castaño. Metasoma castaño oscuro con el terguito 1 más claro.

Hospedadores: Coloradoa bournieri Remaudière \& Leclant, 1969 sobre Santolina chamaeocyparissus L. en Puebla de San Miguel (Valencia): 19-5-93, 3-6-93, 10-6-93; La Yesa (Valencia): 8-695, 28-6-95. Coloradoa moralesi Remaudière \& Leclant, 1969 sobre Santolina rosmarinifolia L. en Viniegra de la Sierra (Rioja): 4-7-95.

Distribución: Provincias de Valencia y Rioja, España.

\section{Discusión}

La especie L. arvensis Stary, 1960, presenta una areola pentagonal, y es un parasitoide específico de diferentes especies de Coloradoa, habiendose citado en Europa sobre C. achilleae H. R.L. y C. bournieri Remaudiere \& Leclant (Stary, 1960; Stary et al., 1971). En España se ha encontrado sobre $C$. bournieri y C. moralesi.

El material estudiado ha sido recolectado siempre sobre santolinas, en el este de la Península Ibérica sobre $S$. chamaecyparissus y en el norte
(Rioja) sobre $S$. rosmarinifolia. La casi totalidad de los ejemplares obtenidos a partir de momias de $C$. moralesi, recogidas sobre $S$. rosmarinifolia, han sido identificados como L. arvensis, mientras que de momias de $C$. bournieri se han separado dos grupos de ejemplares, uno más numeroso que se corresponde con L. arvensis y otro menor que se corresponde con el nuevo taxón descrito.

El nuevo taxón pertenece al grupo de L. adelocarinus, que un estudio reciente (Stary, com. pers., 1996) lo asocia a pulgones de artemisias y que presenta en el propódeo una areola abierta. Aunque los hábitats de Santolina, hasta el momento presente, se encuentran principalmente restringidos a habitats xerocamefíticos, este género, junto con Artemisia, pueden haber invadido vastas áreas durante los diferentes periodos interglaciales pleistocénicos o durante la crisis messiniense. De hecho, se pueden encontrar todavía en la Península Ibérica asociaciones Santolina-Artemisia, por ejemplo en Los Monegros (Zaragoza-Huesca) o en las partes semiáridas de Murcia-Almería, donde diferentes especies de Artemisia (A. herba-alba, A. campestris ssp. glutinosa, A. barrelieri, A. lucentica) coexisten con S. chamaecyparissus (Domínguez et al., en prensa). El desarrollo y expansión de Artemisia sobre vastas estepas durante los periodos geológicos anteriores ha sido ampliamente documentado (Quézel, 1985), y ésto junto con la proximidad química, estructural y taxonómica de ambos géneros explicaría que compartan en muchas ocasiones una entomofauna especializada, como ha sido constatado en otros grupos de insectos (Domínguez et al., en prensa).

De acuerdo con los datos aportados anteriormente, si L. adelocarinus es un parasitoide de pulgones de artemisias, es posible que el nuevo taxón pudiera parasitar también a otros pulgones de artemisia y que $C$. bournieri y $C$. moralesi, sobre los que se ha encontrado, son sólo algunos de los posibles hospedadores, dentro de un rango más amplio.

\section{AGRADECIMIENTOS}

Al Dr. Petr Stary por la información sobre L. adelocarinus y comentarios sobre los ejemplares de comparación enviados. 


\section{Referencias}

Domínguez, M., Oltra, M.T., Baixeras, J. \& YELA, J.L., 1996. Insect herbivore-plant interactions in Mediterranean habitats: Moths associated with Santolina chamaecyparissus and Santolina rosmarinifolia (Asteraceae). $J$. Entomol. Sci.

Mackauer, M., 1968. Hymenopterum Catalogus. Pars 3. Aphidiidae. Dr. Junk N-V. Gravenhage. $103 \mathrm{pp}$.

Smith, C. F., 1944. The Aphidiinae of North America (Braconidae, Hymenoptera). Columbus. The Ohio State Columbus. 154 pp.

QuÉZEL, P., 1985. Definition of the Mediterranean region and the origin of its flora. In: C. GómezCampo (ed.). Plant Conservation in the Mediterranean Area. Junk, Dordrecht: 9-24.

Remaudiere, G. \& Leclant, F., 1969. Deux Coloradoa nouveaux vivant sur Santolina en Europe occidentale (Homoptera, Aphididae). Ann. Soc. Entomol. France (N.S.): 657-668.
StARY, P., 1960. The Aphidiid genus Lysaphidus Smith C.F. in Europe (Hymenoptera, Aphididae). Polskie Pismo Entomologiczne, 30: 359-366.

Stary, P., Remaudiere, G. \& Leclant, F., 1971. Les Aphidiidae (Hymenoptera) de France et leurs hôtes (Homoptera, Aphididae). Entomophaga. Mémoire hors série, $\mathrm{N}^{\mathrm{o}} 5$. Le François Editeur. Paris. 72 pp.
Recibido, el 7-II-1996 Aceptado, el 29-VII-1996 Publicado, el 27-XII-1996 